\title{
Modal Analysis of a Circular Slot Monopole Antenna for UWB application
}

\author{
Adamu Halilu Jabire ${ }^{1,2 *}$, Anas Abdu ${ }^{1}$, Salisu Sani ${ }^{3}$, Sani Saminu ${ }^{4}$, Aliyu Uba Taura ${ }^{1}$, \\ Mohammed Olatunji Obalowu ${ }^{5}$
}

\author{
${ }^{1}$ School of Electronics and Information Engineering, Hebei University of Technology P.R. China. \\ ${ }^{2}$ Department of Electrical and Electronics Engineering, Taraba State University, Jalingo, Taraba State, Nigeria \\ ${ }^{3}$ Department of Electrical Engineering, Ahmadu Bello University Zaria, Kaduna State, Nigeria. \\ ${ }^{4}$ School of Electrical Engineering, Hebei University of Technology, P. R. China. \\ ${ }^{5}$ Department of Electrical and Electronics Engineering, University of Ilorin, Ilorin Nigeria
}

*Corresponding author: adamu.jabire@tsuniversity.edu.ng, Tel: +8615510965699, +2348036570803

\begin{abstract}
In this study, a wideband double slot antenna is introduced. The proposed monopole antenna is designed using the theory of characteristic mode for slot monopoles. A circular shape is used as the initial design stage, to enhance the bandwidth, two circular slots are employed. Four modes have been excited to gain a physical insight and to find out which mode is dominant. The design and analysis were completed utilizing both the time domain and multilayer solver in CST 2017, without considering the feeding port. An antenna model in UWB frequency is constructed. Experimented and simulated results shows that the proposed planar structure has a wide impedance width with good radiation characteristics.
\end{abstract}

Keywords: Characteristic modes (CM), UWB antenna, defected ground structure antenna, monopole antenna.

Article History: received 26 October 2019; accepted 11 December 2019; published 18 December 2019.

(C) 2019 Penerbit UTM Press. All rights reserved

\section{INTRODUCTION}

Designing UWB with concise size and without compromising on antenna performance is a major task nowadays. The UWB systems has become very attractive because of its high transmission data rates and simpler hardware configuration. For wideband operation the circular monopole reception apparatus is a good choice because of the ease of design and construction.

Federal communication commission has dispensed an unlicensed frequency spectrum of $3.1-10.6 \mathrm{GHz}$ for UWB frameworks [1]. The patch antenna, which is a critical component of the modern wireless communication system, has played a vital role in the evolution [2]. Microwave radiators compared with traditional microwave reception apparatus are small, ease, easy to produce and they are effectively coordinated into versatile radio and wireless correspondence applications. The fundamental limitation of microstrip antennas are lower gain and impedance width which can be improved by using metamaterials as part of several techniques [3].

For the most part openings are generally cut over a ground plane or over the emanating component to get a slot reception apparatus. Antenna slot with various types of slot shapes have been developed for many specific applications. For example, circular ring [4], fractal [5], arc [6], triangle [7], T- shape [8] and space shapes like ellipse [9] are proposed to acknowledge wide impedance width. To further gain a deep physical insight into the antenna physical properties and radiation mechanisms the theory of characteristic modes (TCM) has been widely employed in antenna engineering. TCM and its practical applications is presented recently by [10] and [11]. To achieve modal analysis, we have some limited approaches some of which are modal expansion methods [12], and spherical methods [13].

In this letter, modal analysis is developed for a circular monopole reception apparatus. We employed both the time domain solver and multilayer solver in which, the time domain solver is responsible for the S-parameters of the proposed antenna, while the multilayer solver is responsible for the modal analysis of the antenna without considering the feed port. The design and analysis was completed using CST version 2017. To summarize, modal analysis of the proposed structure has been employed to design an antenna suitable for UWB applications. The ampleness of the design strategy is approved through experiment. The remainder of the investigation is sorted out as follows: section 2 introduces the hypothesis behind the modal analysis of antenna, section 3 is the antenna design geometry and parametric analysis of the proposed reception apparatus, section 4 presents the results and discussion of the design and section 5 is the conclusion.

\section{THE THEORY OF CHARACTERISTIC MODE}

Characteristic mode theory was well described in [14 16]. By considering a conducting surface $S$. The total tangential electric field on the body is given by

$$
\left[L(I)-E^{i n}\right]_{\tan }=0
$$


Where $E^{\text {in }}$ is an incident electric field, and L(I) is the dispersed field due to the current I on S. Introducing the notation

$$
Z(I)=[L(I)]_{\tan }
$$

Where $Z$ is the symmetric operator, and setting $Z=R+j X$, the following weighted eigenvalue problem is defined:

$$
Z\left(I_{n}\right)=V_{n} R\left(I_{n}\right)
$$

$V_{n}$ is the eigenvalues, the $I_{n}$ is the modal currents and $R$ is a weight operator. By using $V_{n}=1+j \lambda_{n}$ and cancel the common term $\mathrm{R}\left(\mathrm{I}_{\mathrm{n}}\right)$ in (3) we then obtain

$$
X\left(I_{n}\right)=\lambda_{n} R\left(I_{n}\right)
$$

The mode currents from a weighted orthogonal set over the conductor's surface S. Each modal current, which can be normalized according to radiated unit power.

$$
\left\langle I_{n}^{*}, R I_{n}\right\rangle=1
$$

The current I, on a conducting body can be expanded as

$$
I=\sum_{n=1}^{N} \alpha_{n} I_{n}
$$

Where the $I_{n}$ is the basic functions of the mode current and the $\lambda_{n}$ is the modal expanded coefficients. For an electrically small radiator, only a few modes are required to characterized the antennas behavior. Modes with little $\lambda_{n}$ are good radiators, those with huge $\lambda_{n}$ are poor radiators, while modes with $\lambda_{n}=0$ are at resonance [17] and [18]. The extend of eigenvalues $\lambda_{n}$ thus tells how well a particular mode radiates. The modal significance (Ms) is defined as

$$
M S=\left|\frac{1}{1+j \lambda_{n}}\right|
$$

Which is related to the conducting body's shape, dimension and frequency, but is independent of any specific excitation. Characteristic angle is defined in [19] and [20] as

$$
\theta=180-\tan ^{-1}\left(\lambda_{n}\right)
$$

Equation (8) gives a clear elucidation of how energy is stored in the monopole antenna, when the angle is $180^{\circ}$ the mode resonates, when it is less than $180^{\circ}$ the mode stores magnetic energy and when it is greater than $180^{\circ}$ the mode stores electric energy.

\section{DESIGN STRATEGY}

\subsection{Antenna Geometry}

The geometry of UWB circular reception apparatus is pictured in Figure 1. The receiving wire is simulated utilizing both time domain and multilayer solver analysis in CST version 2017. The system is deployed on a dielectric substrate: FR4 epoxy with an overall permittivity of $4.3,0.025$ loss tangent and stature of $1.6 \mathrm{~mm}$. The absolute structure zone was to improve the impedance width of the proposed receiving wire from $2.8 \mathrm{GHz}$ to $12 \mathrm{GHz}$, two slots are set at each side of the transmitting component with a defected ground plane. The two openings assume an imperative job in improving the impedance width of the proposed reception apparatus. Great portrayal as far as wideband and radiation properties are the benefits of circular shaped reception apparatus. The enhanced elements of the circular formed receiving wire are delineated in Table 1 below. Another structure which we can called it a defective structure has also plays a very important role for improving the performance of the monopole antenna, as clearly illustrated in figure $1 \mathrm{~b}$. The location of the gap controls the bandwidth of the monopole antenna for the fact that, current distribution in the ground will create influences and the behavior of the receiving wire.

The modal analysis was applied to the monopole reception apparatus without feeding port using multilayer solver which represents the standardized amplitude of the modal current. This standardized amplitude doesn't rely upon the feeding port however just relies upon the shape, and size of the conducting object. Another important factor to be consider is the use of loss free substrate instead of lossy parameters because loss free is the prerequisite of using multilayer solver in CST.

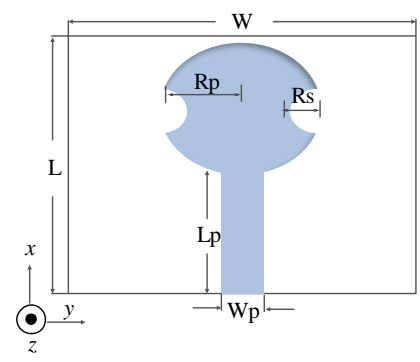

(A)

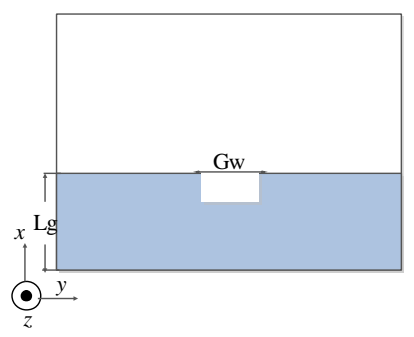

(B)

Figure 1. Circular monopole antenna geometry: A, top layer, B, ground plane

Table 1. Dimension parameters of the proposed antenna

\begin{tabular}{|c|c|c|c|}
\hline Parameter & Size $(\mathrm{mm})$ & Parameter & Size (mm) \\
\hline $\mathrm{L}$ & 30 & $\mathrm{R}_{\mathrm{S}}$ & 2.6 \\
\hline $\mathrm{L}_{\mathrm{g}}$ & 10 & $\mathrm{~W}$ & 30 \\
\hline $\mathrm{L}_{\mathrm{P}}$ & 11.05 & $\mathrm{~W}_{\mathrm{P}}$ & 3 \\
\hline $\mathrm{R}_{\mathrm{P}}$ & 9.35 & $\mathrm{G}_{\mathrm{W}}$ & 3 \\
\hline
\end{tabular}




\subsection{Parametric analysis of the design}

The parametric analysis is presented using different values of $R_{S}$, that is the radius of the slots from Figure 2, in order to have an optimum value that can give us a better performance of the reception apparatus regarding the reflection coefficient, radiation characteristics and bandwidth. Figure 3.6 shows S11 for the various radius of the slot as can be seen from the plot, when the value of $R_{S}$ is $2.6 \mathrm{~mm}$ the resulting $S_{11}$ is perfect at that value. Therefore, we used $2.6 \mathrm{~mm}$ as the radius of the slots for both the two reception apparatus. Even though, the remaining plots are also within the range of $-10 \mathrm{~dB}$, also has a good result but with $2.6 \mathrm{~mm}$ as the value of radius the performance has improved that's is why the value is taken as the final value for the design and analysis of the proposed reception apparatus.

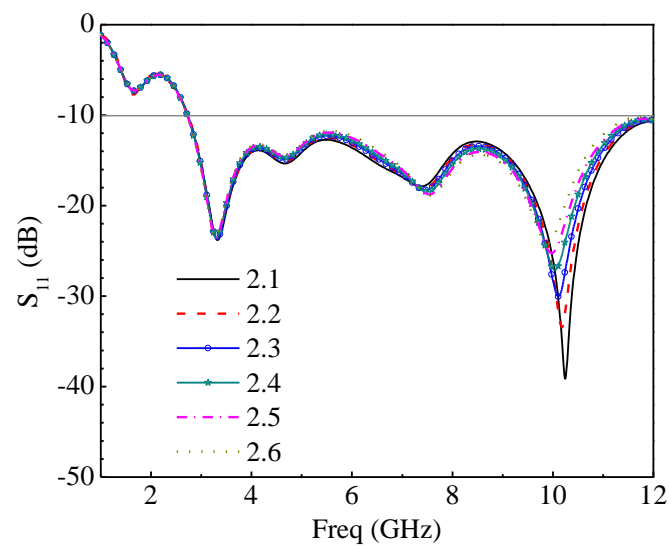

Figure 2. $\mathrm{S}_{11}$ plot for various radius $\mathrm{Rs}(\mathrm{mm})$

\section{RESULTS AND DISCUSSION}

In light of the structure parameters appeared in Figure 1, the single reception apparatus was simulated and analyzed. The construction of the single element and measurement were carried out at the Institute of MicroNano Photo-electronic and Electromagnetic Technology Innovation School of Electronics and Information Engineering, Hebei University of technology, China using N5244A network analyzer. Figure 3 presents the measured and simulated $|\mathrm{S} 11|$ against frequency of the circular monopole antenna, It can be observed from Figure 3 that the $|\mathrm{S} 11|$ is $<-10 \mathrm{~dB}$ with an impedance percentage bandwidth of $200 \%$ from $2.8 \mathrm{GHz}$ to $12 \mathrm{GHz}$. The constructed photo of single element circular shaped antenna is pictured in Figure 4.

The modal significance of the first four modes at $3.35 \mathrm{GHz}$ is presented in Figure 5, only mode 1 has exactly modal significance of up to 1.0. The remaining mode has been shifted a little bit, therefore, only mode 1 will dominate at this frequency response. At $5.5 \mathrm{GHz}$ mode 1 has exactly modal significance of nearly 1.0 , mode 2,3 and 4 is not at $5.5 \mathrm{GHz}$, therefore mode 1 will also dominate at this frequency as shown in Figure 6. The validity of the model further implies that we can consider the antenna shape and feed design as independent steps. Figure 7 presents the characteristic angle for the circular monopole antenna at mode 1, 2, 3 and 4. As mentioned earlier a mode resonates when $\lambda_{n}=0$, that is when the angle is $180^{\circ}$. From the figure mode 1 is at $3.35 \mathrm{GHz}$ and remains the frequency of interest. At $5.5 \mathrm{GHz}$ it is only mode 1 that is at that frequency as shown in Figure 9, the remaining modes have been shifted. It was clearly shown that for all the two frequency of interest the characteristic angle is at $180^{\circ}$ which signifies that the mode is a good radiator.

Figure 10 and Figure 11 presents the eigenvalues for the four modes at $3.35 \mathrm{GHz}$ and $5.5 \mathrm{GHz}$. From the results it clearly shows that the modes are at resonant with $\lambda_{n}=$ 0 but mode 1 is more dominant. Figure 12 is the normalized 2D modal far field pattern at $3.35 \mathrm{GHz}$ for four modes while figure 13 remain the normalized $2 \mathrm{D}$ pattern at $5.5 \mathrm{GHz}$ for mode $1,2,3$ and 4 respectively. The pattern shows the XZ-plane and YZ-planes at the frequency of interest.

Figures 13 to 16 presents the modal surface current distribution and the modal 3D far field pattern of four different modes at $3.35 \mathrm{GHz}$

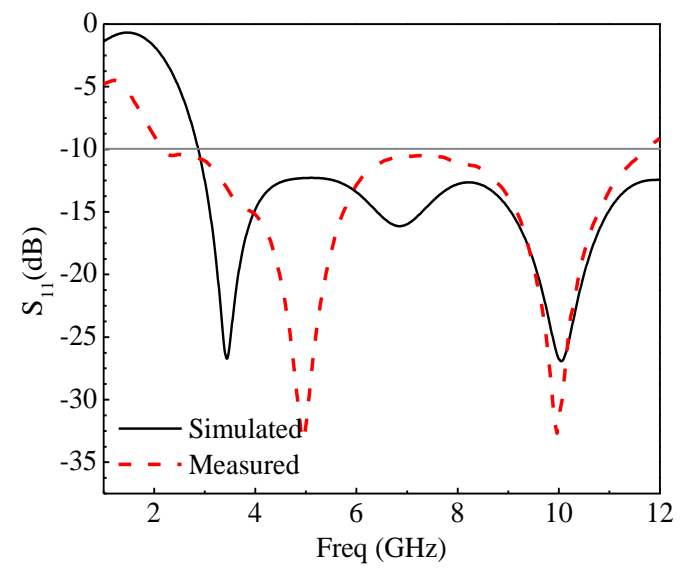

Figure 3. Measured and simulated $S_{11}$ monopole antenna

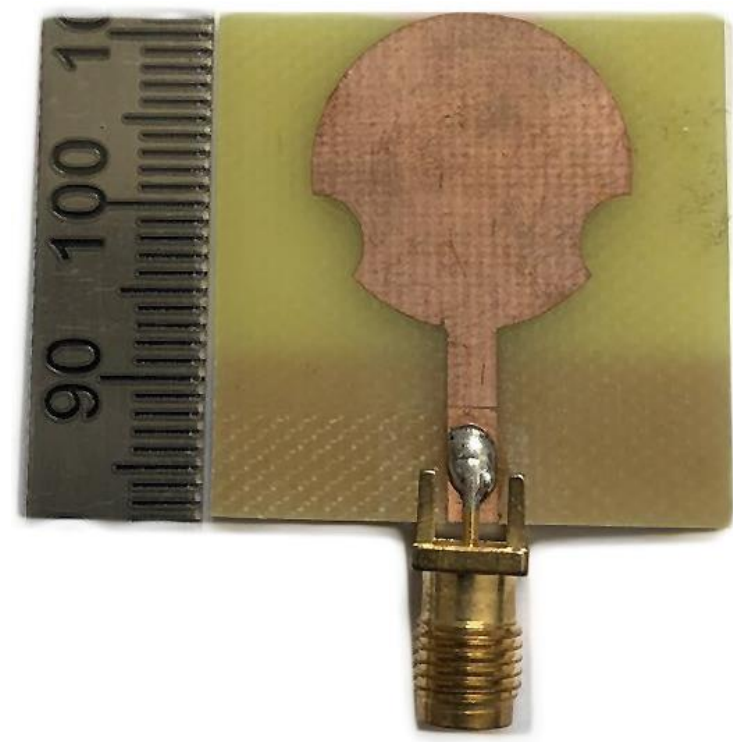

(A) 


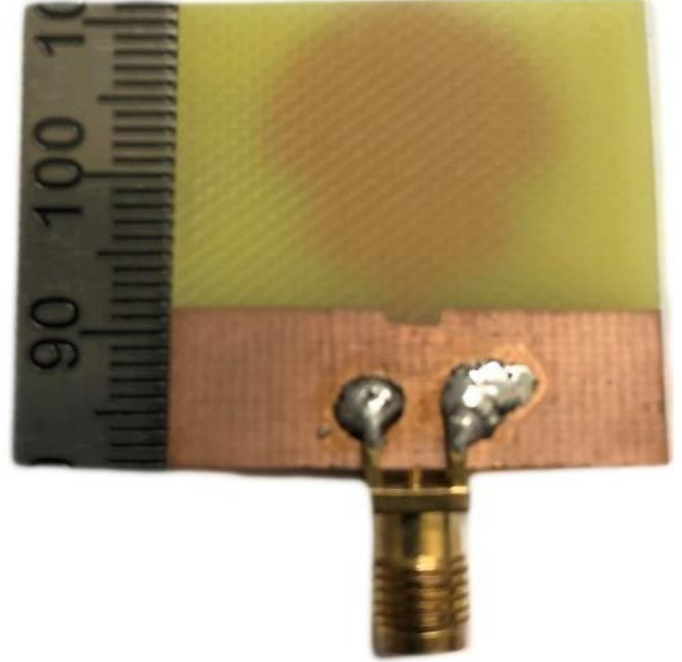

(B)

Figure 4. Fabricated prototype: A, top view, B, bottom view

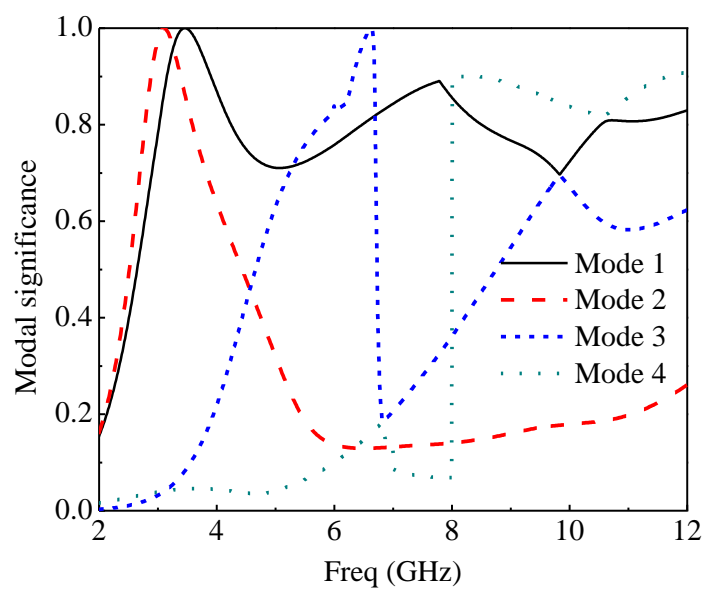

Figure 5. Modal significance at $3.35 \mathrm{GHz}$

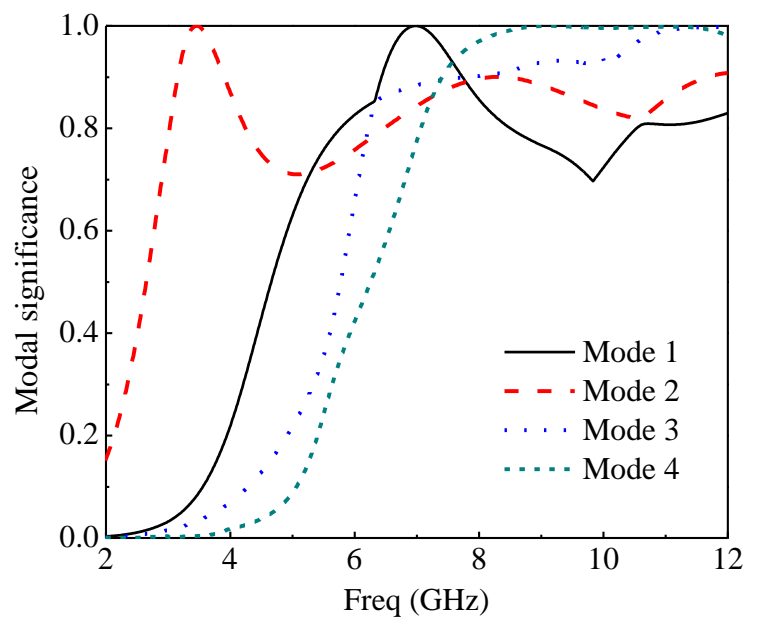

Figure 6. Modal significance at $5.5 \mathrm{GHz}$

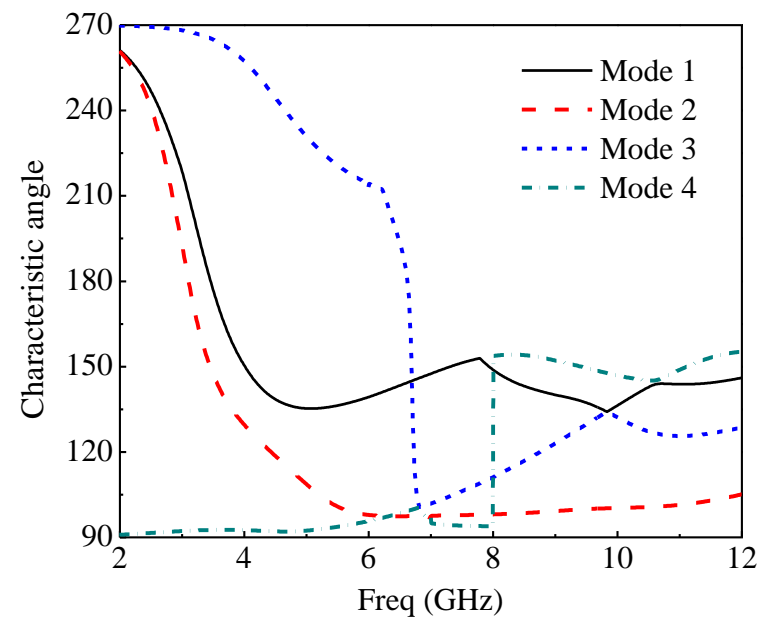

Figure 7. Characteristic angle at $3.35 \mathrm{GHz}$

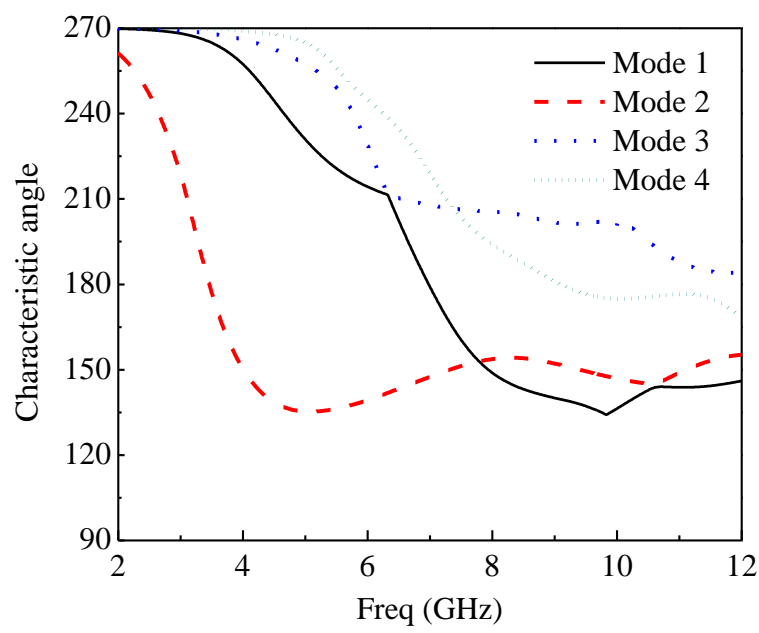

Figure 8. Characteristic angle at $5.5 \mathrm{GHz}$

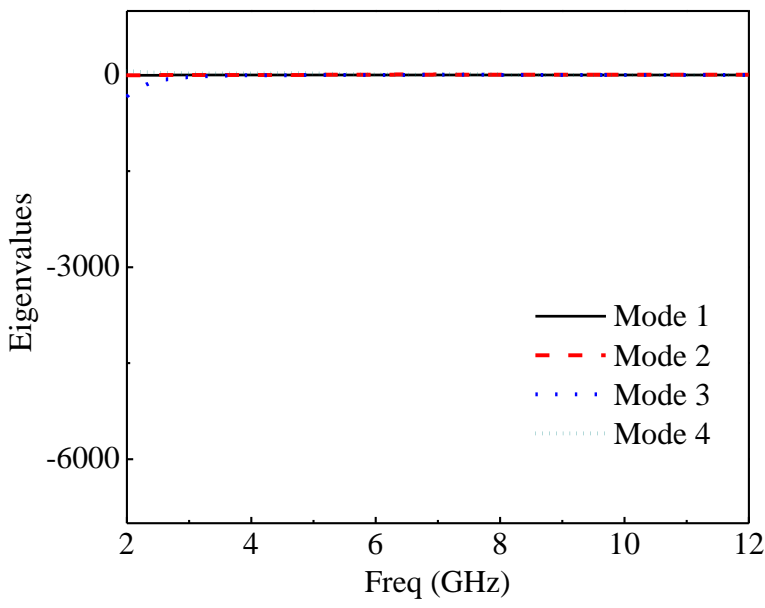

Figure 9. Eigenvalues at $3.35 \mathrm{GHz}$ 


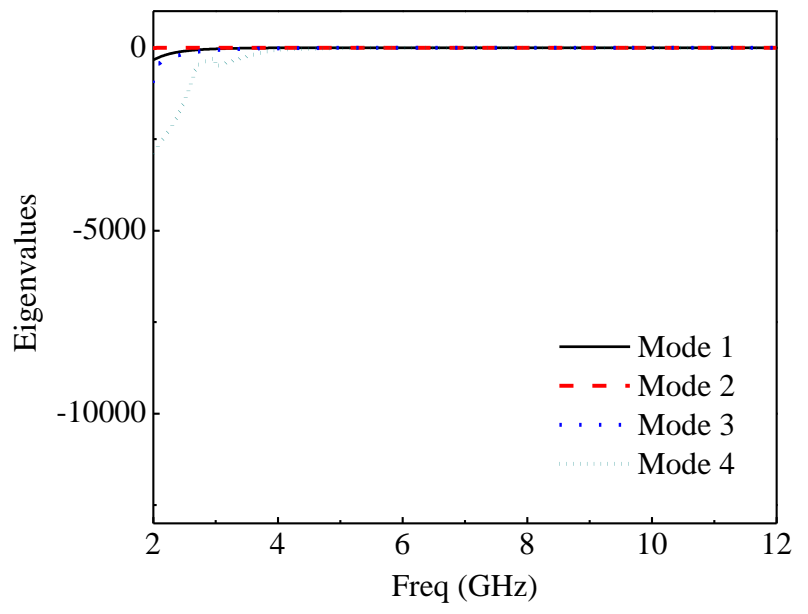

Figure 10. Eigenvalues at $5.5 \mathrm{GHz}$

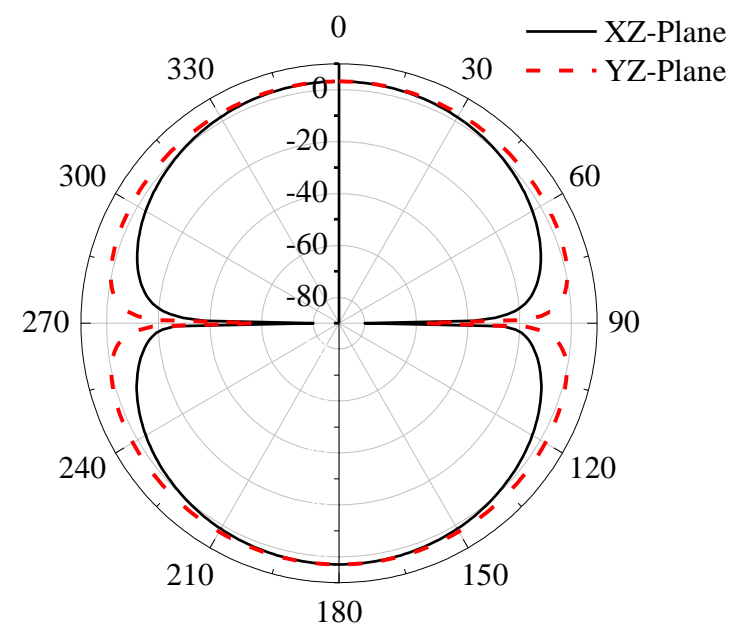

(a)

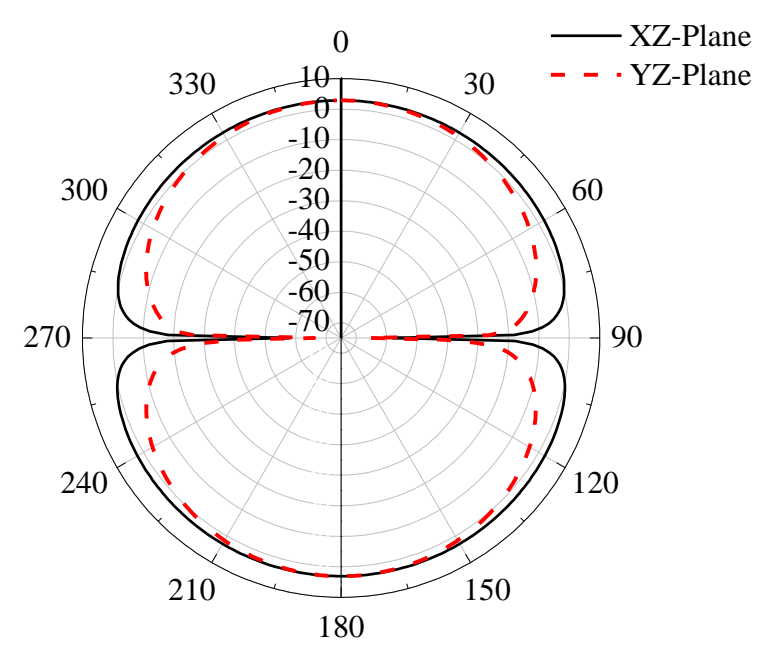

(b)

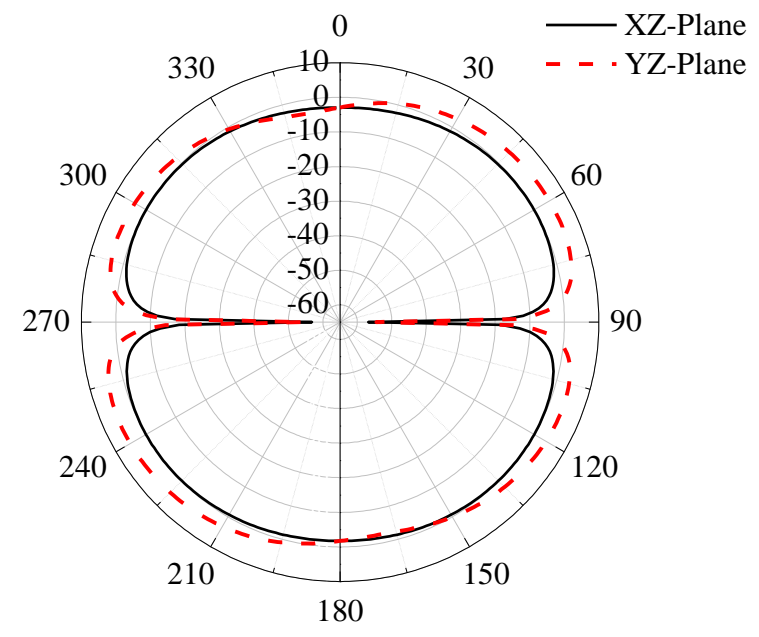

(c)

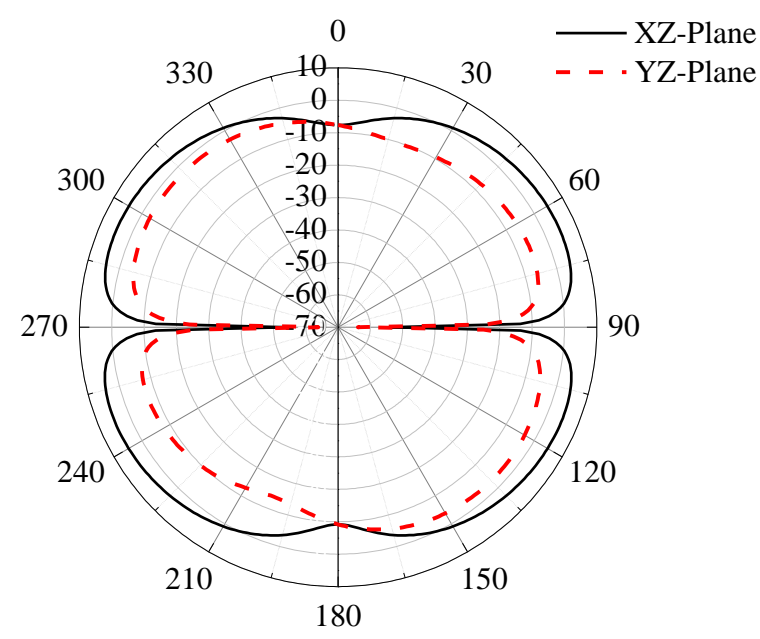

(d)

Figure 11. Far field pattern at $3.35 \mathrm{GHz}$ for (a) mode 1, (b) mode 2, (c) mode 3 and (d) mode 4

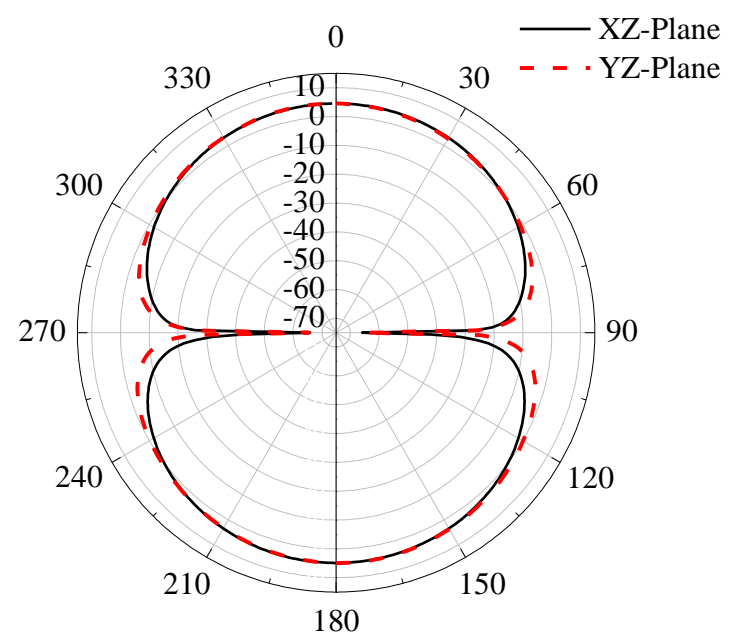

(a) 

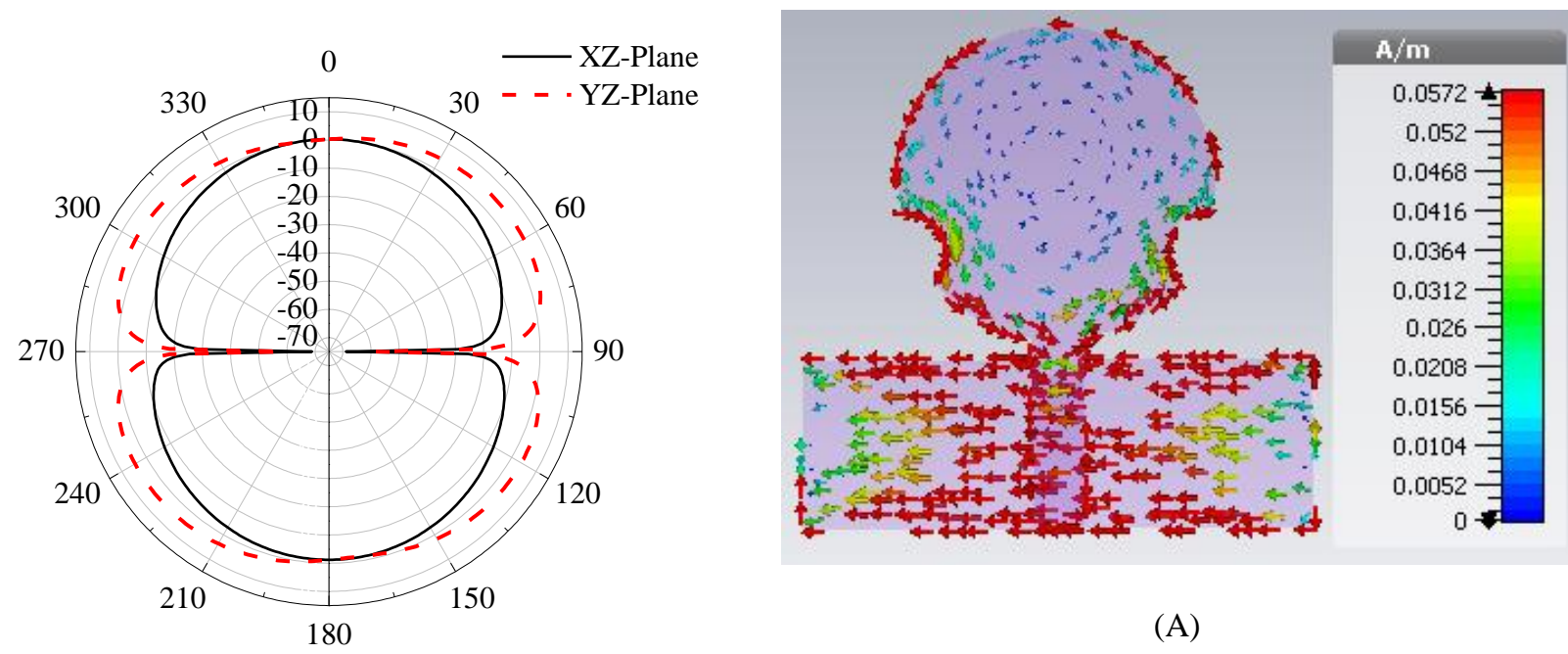

(A)

(b)
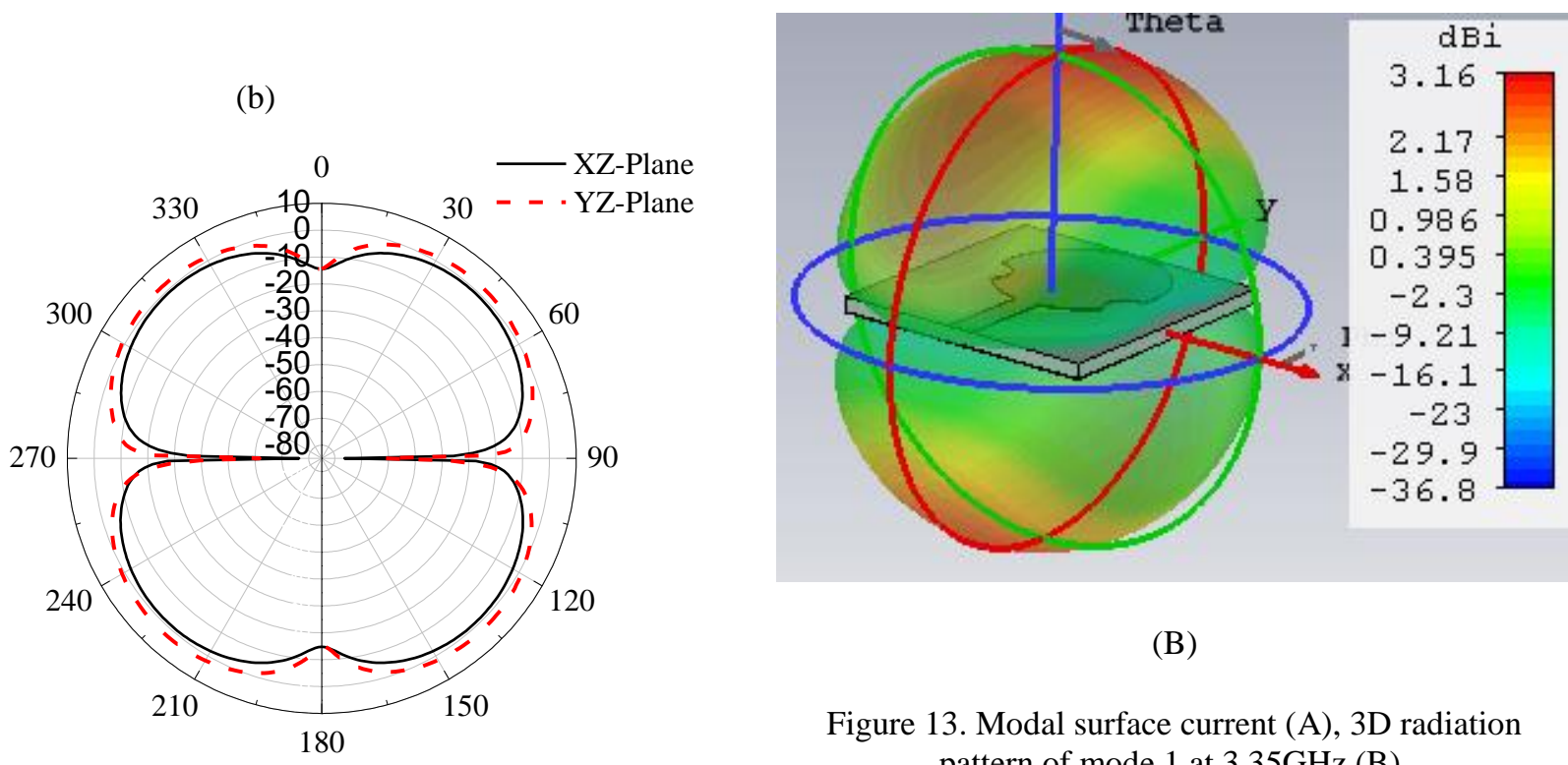

(B)

Figure 13. Modal surface current (A), 3D radiation pattern of mode 1 at $3.35 \mathrm{GHz}(\mathrm{B})$.

(c)
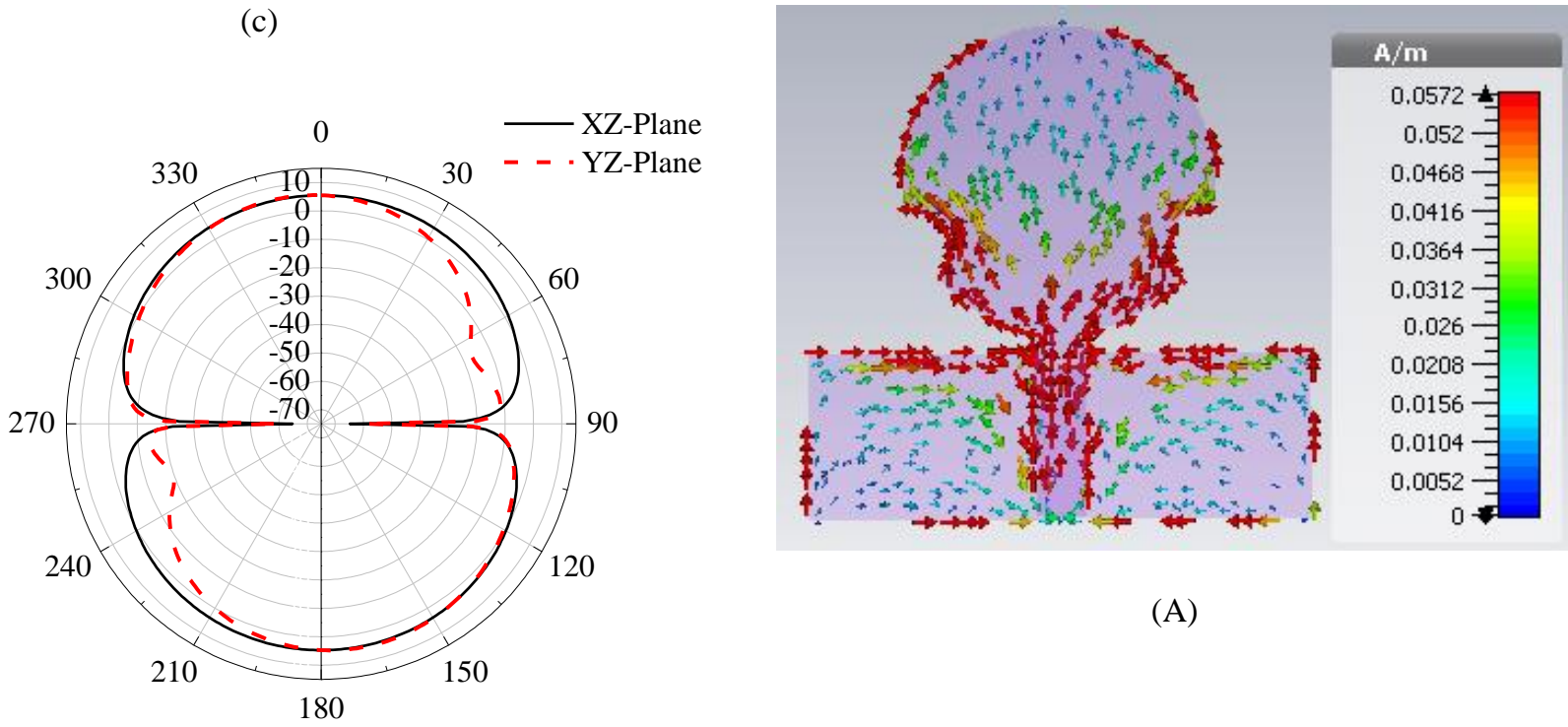

(A)

(d)

Figure 12. Far field pattern at $5.5 \mathrm{GHz}$ for (a) mode 1, (b) mode 2, (c) mode 3 and (d) mode 4 


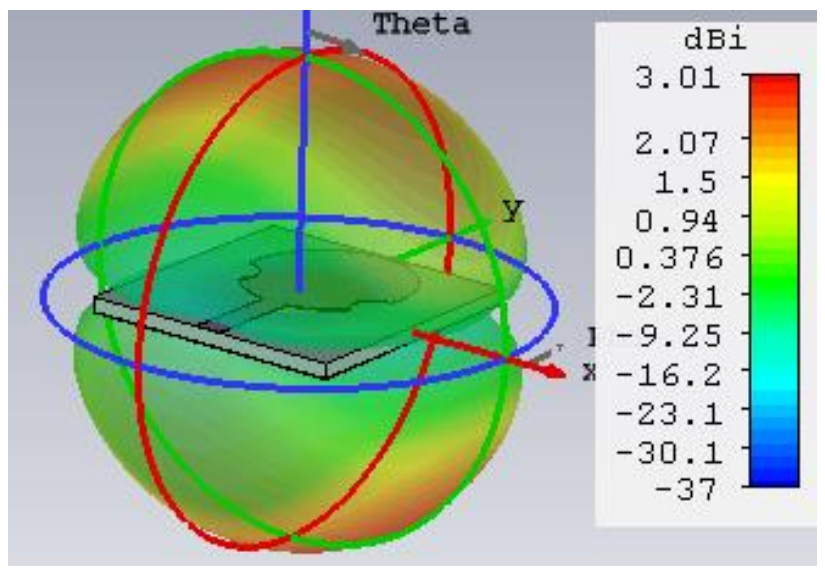

(B)

Figure 14. Modal surface current (A), 3D radiation pattern of mode 2 at $3.35 \mathrm{GHz}(\mathrm{B})$.

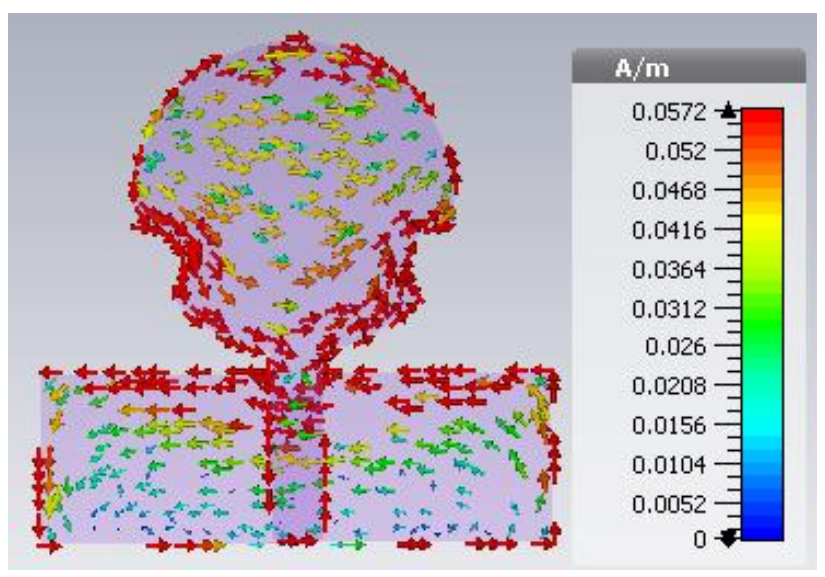

(A)

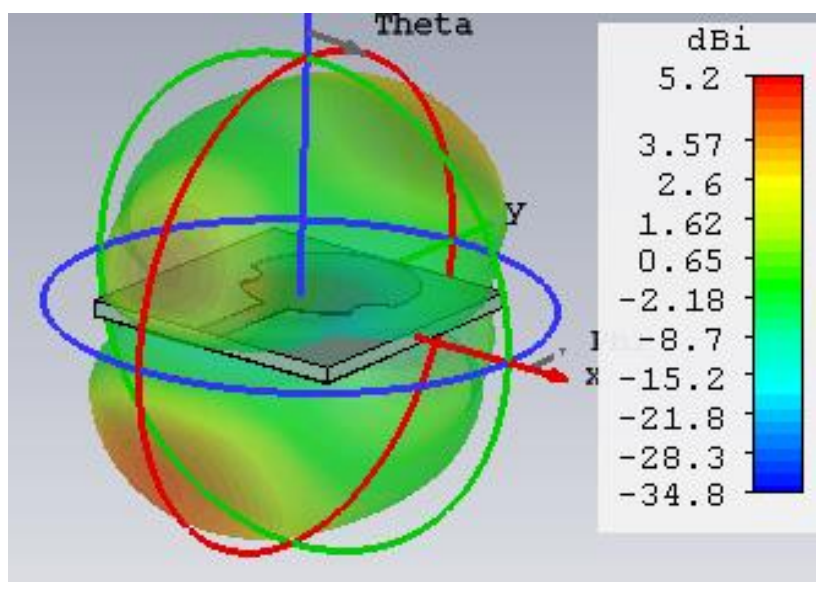

(B)

Figure 15. Modal surface current (A), 3D radiation pattern of mode 3 at $3.35 \mathrm{GHz}(\mathrm{B})$.

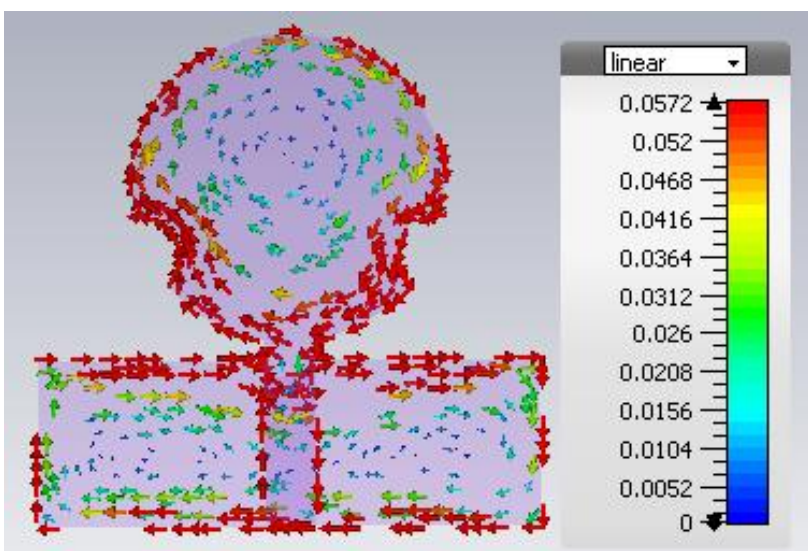

(A)

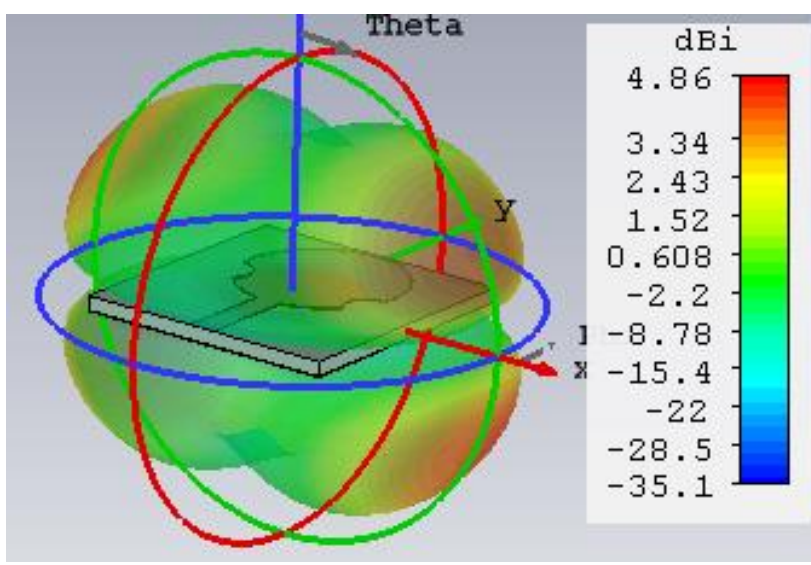

(B)

Figure 16. Modal surface current (A), 3D radiation pattern of mode 4 at $3.35 \mathrm{GHz}$ (B).

Figures 17 to 20 presents the modal surface current distribution and the modal 3D far field pattern of four different modes at $5.5 \mathrm{GHz}$. As can be clearly seen It is obvious that the structure can support more than one mode in the frequency band of interest in two orthogonal directions.

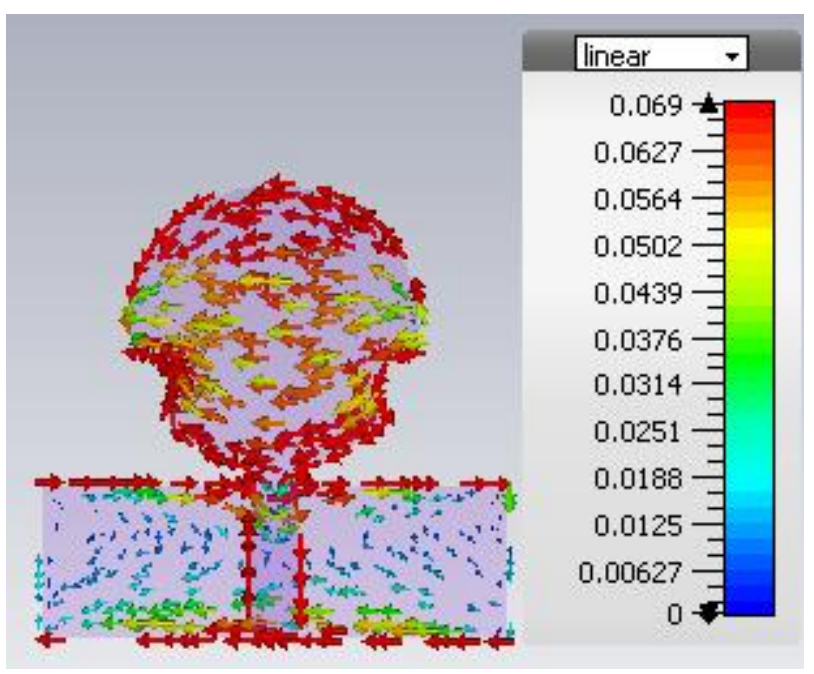

(A) 


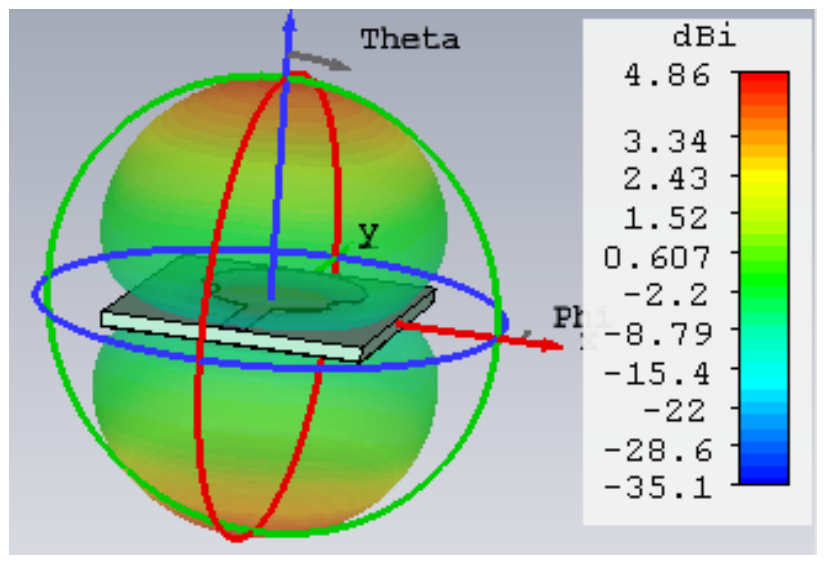

(B)

Figure 17. Modal surface current (A), 3D radiation pattern of mode 1 at $5.5 \mathrm{GHz}(\mathrm{B})$.

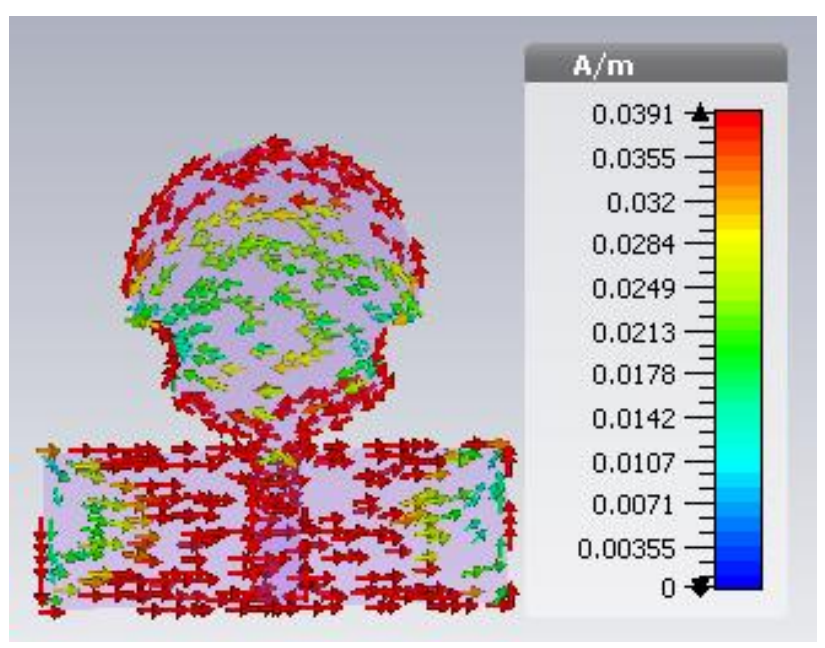

(A)

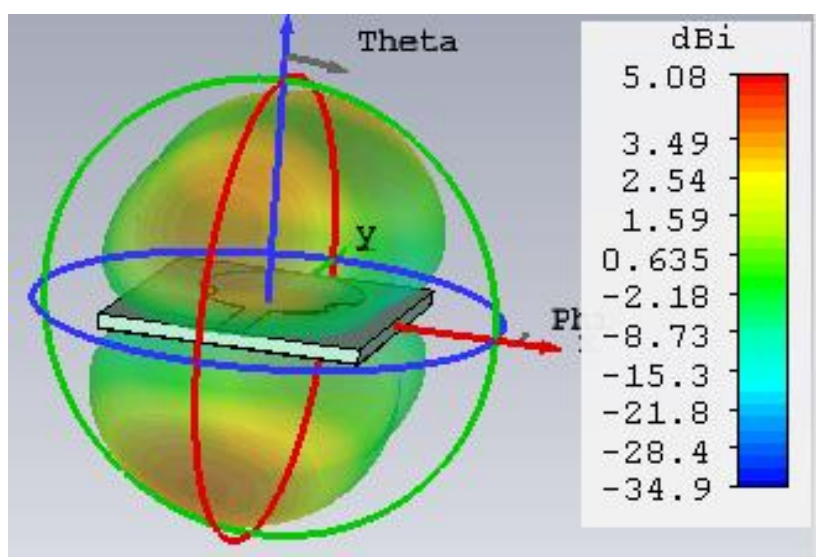

(B)

Figure 18. Modal surface current (A), 3D radiation pattern of mode 2 at $5.5 \mathrm{GHz}(\mathrm{B})$.

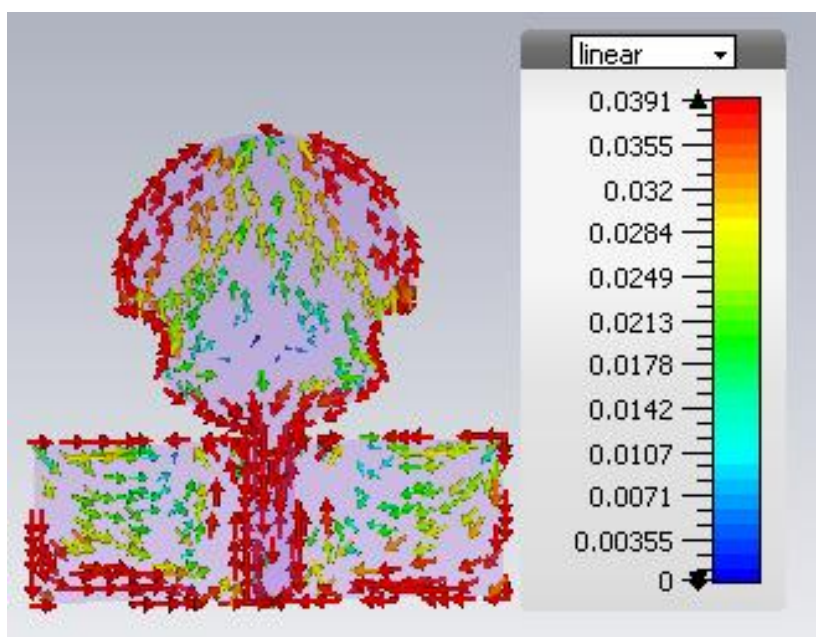

(A)

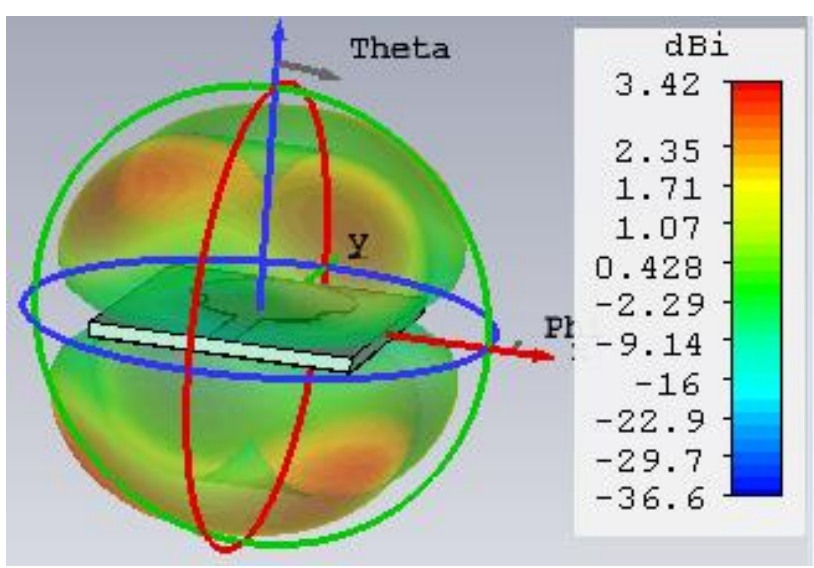

(B)

Figure 19. Modal surface current (A), 3D radiation pattern of mode 3 at $5.5 \mathrm{GHz}(\mathrm{B})$.

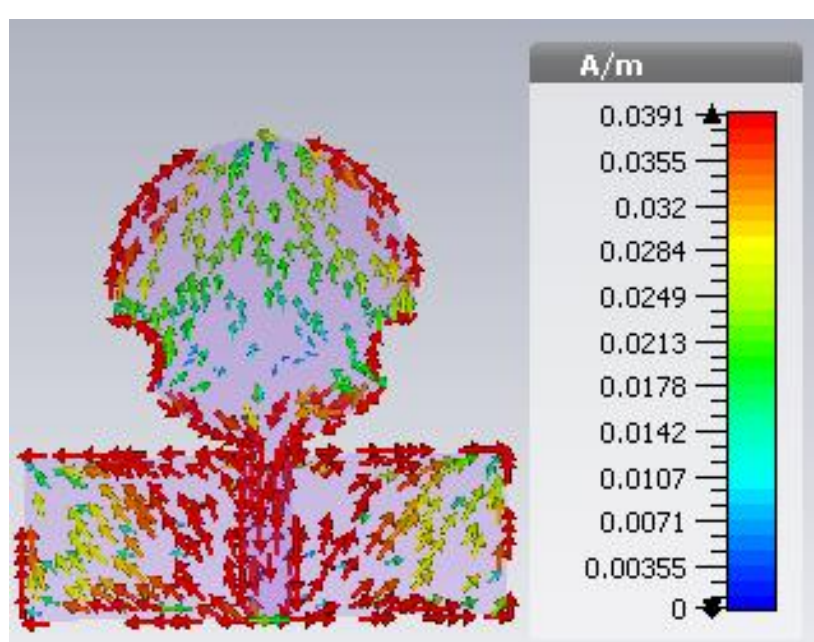

(A) 


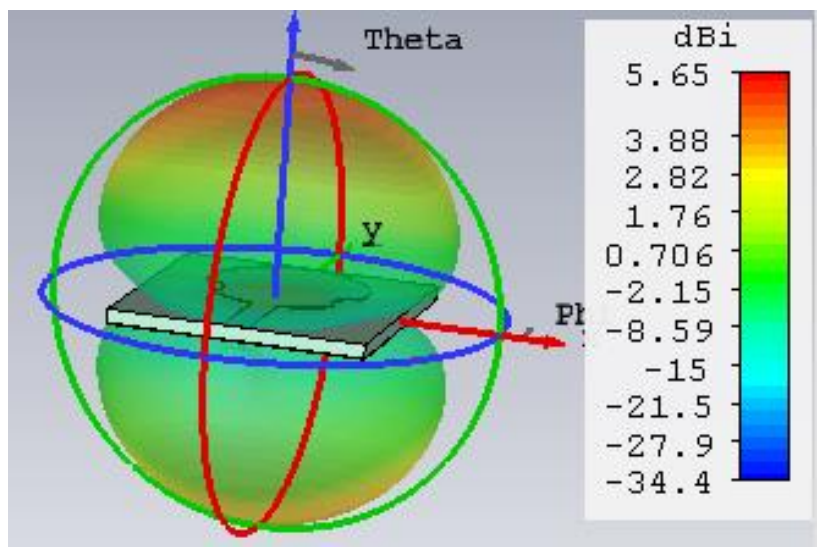

(B)

Figure 20. Modal surface current (A), 3D radiation pattern of mode 4 at $5.5 \mathrm{GHz}(\mathrm{B})$.

The bandwidth of the proposed receiving wire is contrasted with past works in Table 2. The proposed circular monopole antenna achieves a considerable wide impedance width and smaller size as compared with trinotched UWB antenna [4], fractal UWB MIMO antenna [5] and circularly polarized slot antenna [6].

Table 2. Comparison between the reference antenna

\begin{tabular}{|c|c|c|}
\hline Reference & Bandwidth (GHz) & Size $(\mathrm{mm})$ \\
\hline$[4]$ & $3.1-11$ & $34 \times 36 \times 1$ \\
\hline$[5]$ & $3.1-12.5$ & $24 \times 32 \times 0.8$ \\
\hline$[6]$ & $2.1-5.6$ & $42 \times 42 \times 1.5$ \\
\hline$[7]$ & $2.9-19.2$ & $40 \times 40 \times 1.5$ \\
\hline Prop. & $2.6-12$ & $30 \times 30 \times 1.6$ \\
\hline
\end{tabular}

\section{CONCLUSION}

The CMA has been applied to a circular monopole antenna using multilayer solver analysis in CST version 2017. The modal significance clearly shows that mode 1 is the dominant mode. The design procedure also removes the additional burden that may arise as a result of feeding port. A prototype has been constructed. Sparameters have been measured to validate the design. Owing to the wide $\mathrm{BW}$, the proposed antenna finds wide application in UWB communication systems.

\section{ACKNOWLEDGMENT}

The authors will like to thank the Institute of Micro-Nano Electronic and Electromagnetic Technology Innovation School of Electronics and Information Engineering, Hebei University of Technology P.R. China.

\section{REFERENCES}

[1] R. N. Tiwari, P. Singh and B. K. Kumar, "Smallsize scarecrow-shaped CPW and microstrip-line fed UWB antennas," Journal of comput electr., vol. 17, pp.1047-1055, 2018.
[2] Ehab K. I. Hamad and Ahmed Abdelaziz, "MTM superstrate microstrip patch antenna for $5 \mathrm{G}$ wireless communication based on the theory of characteristics modes," Journal of Electrical Engineering, vol. 70(3): pp.187 - 197, 2019.

[3] A. H. Jabire, H-X Zheng, A. Abdu et al, "Characteristic mode analysis and design of wide band MIMO antenna consisting of metamaterial unit cell," Electronics, vol. 8(1), pp.1-14, 2019.

[4] S. Rahman, Q. Cao, Y. Li, et al, "Design of trinotched UWB antenna based on elliptical and circular ring resonators," Int. J. RF Microw Comput Aided Eng, vol. 29(3): e21632, 2019.

[5] Gurjar R, Upadhyay DK, Kanaijia BK, Sharma K, "A novel compact self-similar fractal UWB MIMO antenna," Int. J. RF Microw Comput Aided Eng, e21632, 2018.

[6] Huy Hung T. Nghia, Nguyen T, and Amin Abbosh M. "Simple design procedure of a broadband circularly polarized slot monopole antenna assisted by characteristic mode analysis," IEEE Access, vol. 6, pp. 78386-78393, 2018.

[7] A. Boutejdar and W. Abdellatif, "A novel compact UWB monopole antenna with enhanced bandwidth using triangular defected microstrip structure and stepped cut technique," Microw and Optical Technology Lett., vol. 58(6): pp. 1514-1519, 2016.

[8] A. H. Jabire, A. Abdu and S. Salisu, "Multiband millimeter wave T-shaped antenna with optimized patch parameters using particle swarm optimization," Nigerian Journal of Technology (NIJOTECH), vol. 36(3): pp. 904-909, 2017.

[9] Kumar R, and Pazare N, "A printed semi-circular disc UWB MIMO/diversity antenna with cross shape slot stub," Wireless Personal Communication, vol. 91(1): pp. 277- 291, 2016.

[10] Z. Ma, Z. Yang, Q. Wu et al. "Out of band mutual coupling suppression for microstrip antennas using characteristic mode analysis and shorting pins," IEEE Access, vol. 7, pp. 102679-102688, 2019.

[11] Feng H. Lin and Z. N. Chen, "Low profile wideband metasurface antennas using characteristic mode analysis," IEEE Trans Antennas and Propag, vol. 65(4): pp. 1706-1713, 2017.

[12] L. Kuang, S. Zhu, J. Gao et al, "A numerical method for analyzing electromagnetic scattering properties of a moving conducting objects," Int. J. Antennas and Propag, vol. 2014, pp. 1-9, 2014.

[13] R. F. Harrington, "Effect of antenna size on gain, bandwidth and efficiency," J. Res. Nat. Bur. Stand. D. Radio Propag, vol. 64D, pp. 1-12, 1960.

[14] Ali A, and Gholamreza D. "Oriented design of an antenna for MIMO applications using theory of characteristic modes," IEEE Antennas and Wirel. Propag. Letters, vol. 11, pp. 1040- 1043, 2012.

[15] R. F. Harrington and J. R. Mautz, "Theory of characteristic modes for conducting bodies," IEEE Trans Antennas Propag, vol. 19, pp. 622-628, 1971.

[16] Chen Y, Wang C. F. "Charactristic modes: theory and application in antenna engineering," Wiley, Hoboken, NJ. USA, 2015.

[17] Liang P. Wu, Q. "Characteristic mode analysis of antenna mutual coupling in the near field," IEEE Antennas Propag, vol. 66, pp. 3757-3762, 2018. 
[18] Kumar Kishor K, and Victor Kumar S. "Multiport multiband chassis-mode antenna design using characteristic modes,"IEEE aAntenna Wireless Propag. Letters, vol. 16, pp. 609-612, 2017.
[19] Li, K. Shi Y. "A pattern reconfigurable MIMO antenna design using chacteristic modes," IEEE Access, vol. 6, pp. 43526-43534, 2018.

[20] Garbacz, R. J. A generalized expansion for radiated scattered fields, phD. Thesis, Ohio University, Columbus, OH. USA, 1968. 